\title{
Universality classes for critical wetting
}

\author{
D. M. Kroll \\ Institut für Festkörperforschung der Kernforschungsanlage Jülich, \\ Postfach 1913, D-5170 Jülich, West Germany \\ R. Lipowsky* \\ Baker Laboratory, Cornell University, Ithaca, New York 14853
}

R. K. P. Zia

Physics Department, Virginia Polytechnic Institute and State University, Blacksburg, Virginia 24061

(Received 4 March 85)

\begin{abstract}
The systematics of the wetting transition at solid-gas interfaces in systems interacting with inversepower-law potentials is investigated. The correct form of the effective Hamiltonian for $l$, the interfacesubstrate separation, is determined, and it is shown that for a given type of continuous wetting transition (e.g., critical wetting) there are three regimes of critical behavior, depending on the spatial dimension $d$. New results for the critical exponents in the intermediate regime are also presented.
\end{abstract}

The nature of the various types of wetting transitions for a given form of interaction potential is still somewhat controversial. ${ }^{1-6}$ In particular, the systematics of these transitions for inverse-power-law potentials is still an open question. $^{4(b), 7-12}$ In this Rapid Communication we investigate this problem for coarse-grained order-parameter interaction potentials of the general form $w(r) \sim r^{-(d+\sigma)}$ as $r \rightarrow \infty$, with $\sigma>1$ where $d$ is the spatial dimension. After establishing the existence of stable mean-field (MF) profiles for $\sigma>1$ we determine the correct form of the effective Hamiltonian for $l$, the interface-substrate separation. It is then shown that there is a sequence of upper critical dimensions (UCD) in the problem and that for a given type of critical or multicritical transition three distinct types of behavior are possible, depending on the spatial dimension $d$. Denoting the $j$ th UCD by $d_{j}, j=0,1, \ldots$, we show that for the potential $V(l)$ given in (3b) below, $d_{j}$ is the spatial dimension where the operator $l^{-(\sigma+j-1)}$ is marginal. In particular, for the critical wetting transition we find that (i) MF theory is correct for $d>d_{1},{ }^{13}$ (ii) for $d_{1}>d>d_{0}$ fluctuations are strong enough to renormalize the exponents but there is no shift of the MF wetting temperature, and (iii) for $d_{0}>d$ the wetting temperature is renormalized and all wetting transitions (including those described by MF theory to be first order) are continuous and belong to the same universality class. A similar sequencing occurs for all multicritical transitions. New results for the critical exponents in the intermediate dimension interval described in (ii) above are also derived and it is argued that for all $d \leqslant 3, \eta$, the anomalous dimension of the interface degree of freedom $l$ is zero. Finally, these effects are illustrated by exact results for $d=2$.

The model we consider is described by the free-energy functional

$$
\begin{aligned}
F\{\phi\}= & \frac{1}{2} \int_{r} \int_{r^{\prime}} \phi(r) w\left(r-r^{\prime}\right) \phi\left(r^{\prime}\right) \\
& +\int_{r}[U(\phi(r))+h(r) \phi(r)-\mu \phi(r)] .
\end{aligned}
$$

The order parameter $\phi$ is generally the density. $w$ is the in- teraction potential, $h$ an external (substrate) potential (derived from an interaction potential with the same asymptotics as $w), \mu$ the chemical potential, and $U(\phi)$ the reference system free-energy density in the van der Waals picture. ${ }^{10}$ This model describes not only wetting at a solid surface but also wetting in binary mixtures to the extent it can be described by a single order-parameter model. For the usual wetting geometries the solutions of interest vary only in one direction, here taken to be $z$. These solutions interpolate between two or more bulk phases. In particular, we want to establish the range of potentials for which (1) has stable MF solutions which fulfill this condition.

Consider first an infinite system at two-phase coexistence ( $h=0$ and $\mu=\mu_{c}$ ). Taking the Fourier transform of (1) we have

$F\{\phi\}=\frac{1}{2} \int_{q} \phi_{q} u_{2}(q) \phi_{-q}+\int_{r} \bar{U}(\phi)=E_{T}\{\phi\}+E_{\bar{U}}\{\phi\}$,

where the $q=0$ part of the Fourier transform of $w(r)$ has been included in $\bar{U}(\phi)$ so that, to leading order, $u_{2}(q)=j_{\pi} q^{\pi}+\cdots$ with $\pi=\min (2, \sigma)$ (Ref. 14) for $\sigma \neq 2$. For $\sigma=2$ there is an additional logarithmic correction term $q^{2}|\ln q|$ in $u_{2}(q) . \quad q$ is the $d$-dimensional momentum conjugate to $r$ and $j_{\pi}$ is positive so that the kinetic part $E_{T}\{\phi\}$ is non-negative. Assume that $\phi_{c}(z)$ is an extremal solution of (2) describing a free interface between two coexisting phases at some arbitrary position $z_{0}$ and define $\phi_{a}(z)=\phi_{c}(z / a)$ where $a$ is a positive parameter. In order that $\phi_{c}(z)$ be a classical minimum of (2), $F\left(\phi_{a}\right)$ must be stationary and $d^{2} F /\left.d a^{2}\right|_{\phi_{a}}>0$ for $a=1$. Performing a change of variables we find that $F\left(\phi_{a}\right)=a^{1-\pi} E_{T}\left(\phi_{c}\right)$ $+a E_{U}\left(\phi_{c}\right) \cdot{ }^{15}$ It follows that

$$
\left.\frac{d^{2} F\left(\phi_{a}\right)}{d a^{2}}\right|_{a=1}=\pi(\pi-1) E_{T}\left(\phi_{c}\right),
$$

so that $F\left(\phi_{c}\right)$ will be a minimum only for $\sigma>1$. Stable MF solutions of finite energy cannot exist ${ }^{16}$ for $\sigma \leqslant 1$.

In order to go beyond MF theory it is necessary to determine the spectrum of fluctuations about the MF profile 
$\phi_{c}(z){ }^{2,17,18}$ This leads to the eigenvalue problem

$$
\left.\int_{\rho^{\prime}} \int_{z^{\prime}} \frac{\delta^{2} F}{\delta \phi(\mathbf{r}) \delta \phi\left(\mathbf{r}^{\prime}\right)}\right|_{\phi_{c}} \psi\left(\rho^{\prime}, z^{\prime}\right)=\epsilon \psi(\rho, z),
$$

where $\rho$ is the $(d-1)$-dimensional spatial coordinate perpendicular to $z$.

Let $\rho$ be the momentum conjugate to $p$ and $q=(p, k)$. Taking the Fourier transform in $\rho$, we obtain the eigenvalue problem

$j_{\pi} \int_{k} \int_{z^{\prime}} \exp \left[i k\left(z-z^{\prime}\right)\right]\left(k^{2}+p^{2}\right)^{\pi / 2} \eta\left(z^{\prime}\right)$

$$
+\bar{U}^{\prime \prime}\left(\phi_{c}(z)\right) \eta(z)=\epsilon_{p} \eta(z),
$$

where $\Psi(\rho, z)=e^{i \rho \cdot p} \eta(z)$. For $p=0$ this eigenvalue problem has a ground-state eigenfunction

$$
\eta(z)=\bar{\eta}(z) \equiv \phi_{c}^{\prime}(z) /\left\|\phi_{c}^{\prime}\right\|,
$$

where

$$
\left\|\phi_{c}^{\prime}\right\|=\left(\int_{z}\left[\phi_{c}^{\prime}(z)\right]^{2}\right)^{1 / 2},
$$

with eigenvalue $\epsilon_{p}=0=0 .{ }^{18}$ It follows that

$$
\epsilon_{p}=j_{\pi} \int_{k} \bar{\eta}_{k}\left[\left(k^{2}+p^{2}\right)^{\pi / 2}-k^{\pi}\right] \bar{\eta}_{-k}
$$

Since $\bar{\eta}_{k=0}$ is finite we find $\epsilon_{p} \sim p^{2}+O\left(p^{1+\sigma}\right)$ for $\sigma>1$, i.e., the allowed range of $\sigma$. The kinetic part of the effective Hamiltonian for the interface degree of freedom $l(\rho)$ is therefore not affected by the long-range nature of the forces. The potential part is affected, however, and is in fact given by the local MF free-energy density of a rigid interface located at height $l{ }^{2,17}$ The effective Hamiltonian is thus

$\mathscr{H}=\frac{\bar{\sigma}}{2} \int_{p} l_{p} p^{2} l_{-p}+\int_{\rho} V(l)=\int_{\rho}\left[\frac{1}{2} \bar{\sigma}(\nabla l)^{2}+V(l)\right]$

to leading order, where $\bar{\sigma}$ is the surface tension of the free interface.

Recent MF results for inverse-power potentials indicate that, ${ }^{10-12}$ for large $l$,

$$
V(l)=a_{0} / l^{r_{0}}+a_{1} / l^{r_{1}}+a_{2} / l^{r_{2}}+\cdots+\Delta \mu l,
$$

where $r_{0}=\sigma-1$ and $r_{i+1}=r_{i}+1$ for $i \geqslant 0 . \quad \Delta \mu=\mu_{c}-\mu$ measures the distance from coexistence. Critical wetting occurs for $\Delta \mu=0, a_{1}>0, a_{0}<0$ as $a_{0} \rightarrow 0^{-} ; a_{0}$ thus plays the role of the reduced temperature. First-order wetting occurs if $a_{1}$, or the coefficient of a higher-order term, is negative and $a_{0}$ is positive. Higher-order multicritical transitions can occur if, for example, $a_{2}>0, a_{1}=0$, and $a_{0}<0$ as $a_{0} \rightarrow 0^{-}$(a tricritical transition). A tetracritical transition occurs for $a_{3}>0, a_{2}=a_{1}=0$, and $a_{0} \rightarrow 0^{-}$, etc. $^{12}$

Near the critical wetting transition the interface freeenergy density has the scaling form ${ }^{19,20} F_{s}=t^{2-\alpha_{s}} \Omega\left(\Delta \mu / t^{\Delta}\right)$. For $V(l)$ given in (3b) the MF approximation yields $2-\alpha_{s}=\sigma$ and $\Delta=1+\sigma$ where $t=\left|a_{0}\right|$. Near the tricritical point ${ }^{19,20} \quad F_{s}=t^{2-\alpha_{s}} \Omega\left(a_{1} / t^{\Delta_{1}}, \Delta \mu / t^{\Delta}\right)$ where $2-\alpha_{s}$ $=\frac{1}{2}(1+\sigma), \Delta_{1}=\frac{1}{2}$, and $\Delta=\frac{1}{2}(\sigma+2)$ in the MF approximation.

Consider now the effect of fluctuations. We start by reviewing the arguments leading to a generalized Ginzburg criterion for the UCD for the model described by (3a) and (3b). Expand $V(l)$ about the MF value of $\langle l\rangle \equiv\langle l\rangle_{0}$. A perturbation expansion for $\Gamma^{(2)}(p=0)$, the two-point vertex function at zero momentum, then yields

$$
\Gamma^{(2)}(0)=\xi^{-2}=\xi_{0}^{-2}+c t \xi_{0}^{3-d}\langle l\rangle_{0}^{-\sigma-3}
$$

to leading order, where $c$ is a constant, $\xi_{0}$ the MF correlation length, and $\xi_{0}^{3-d}$ the infrared divergence of the relevant diagram. MF theory is valid if $\xi_{0}^{-2}$ $\gg t \xi 0^{-d}\langle l\rangle_{0}^{-\sigma-3}$. Using the index $j$ to number the various types of transitions ( $j=1$ critical, $j=2$ tricritical, etc.), this implies that the UCD for transition $j$ is $d_{j}=[3(\sigma+j)-1] /(\sigma+j+1) . \quad d_{j}$ is the spatial dimension where the operator $l^{-\sigma-j+1}$ is marginal.

There is a simple way of understanding the Ginzburg criterion. The mean-square width of the interfacial region is $\left\langle[l-\langle l\rangle]^{2}\right\rangle=G(0)$ where $G(\rho)=\langle l(0) l(\rho)\rangle-\langle l\rangle^{2}$. Below the wetting transition, for finite $\xi, \xi_{1}^{2} \equiv G(0) \sim \xi^{3-d}$ (since $\eta=0$ in MF theory). ${ }^{7}$ Requiring $\langle l\rangle=\xi_{\perp}$ implies $\beta_{s}=\frac{1}{2}(d-3) \nu$, which is just the hyperscaling relation. This scaling relation is fulfilled by the MF exponents for transition $j$ at $d_{j} .{ }^{5}$ For $d>d_{j}$, MF theory implies $\langle l\rangle>\xi_{\perp}$ at transition $j$. In the case of wetting at a solid-gas interface this means that the mean distance of the interface from the substrate is greater than the rms width of the interface so that fluctuations cannot influence the transition. Below $d_{j}$ the opposite is the case. Fluctuations are responsible for driving the wetting transition: $\langle l\rangle$ is determined by the rms size of interface fluctuations.

Before discussing corrections to MF behavior, it is important to note that for all $d \leqslant 3, \eta$, the anomalous dimension of $l$, should be zero. ${ }^{21}$. Define $g(\rho)=\left\langle[l(\rho)-l(0)]^{2}\right\rangle$ $=2[G(0)-G(\rho)]$. Capillary-wave theory indicates that above the transition $g(\rho) \sim \rho^{3-d}$ for $d<3$ and $g(\rho) \sim \ln \rho$ for $d=3 .^{22}$ The general scaling form for $G(\rho)$ below the transition is $G(\rho)=\xi^{3-d-\eta} \Phi(\rho / \xi)$. However, this expression is compatible with the capillary-wave picture above $T_{w}$ only if $\eta=0$. In this case, $g(\rho) \sim \xi^{3-d}[1-\Phi(\rho / \xi)]$. Analyticity at small $\rho / \xi$ implies $\Phi(x) \sim 1+x+\cdots$ for $x \rightarrow 0$ so that $g(\rho) \sim \rho^{3-d}$ for $d<3$ and $g(\rho) \sim \ln \rho$ for $d=3$, in agreement with capillary-wave theory. This result together with standard scaling laws then implies $\beta_{s} / \Delta=(d-3) /(d+1)$ so that $F_{s} \sim(\Delta \mu)^{2(d-1) /(d+1)}$ and $\langle l\rangle \sim(\Delta \mu)^{(d-3) /(d+1)}$ at all continuous transitions $j$ when $d<d_{j}$. The $\Delta-\eta$ dependence is universal.

It turns out that it is rather easy to obtain the critical exponents for all multicritical transitions $j$ in the dimension interval $d_{j-1}<d<d_{j}$. The important vertices are those obtained from the term $a_{0} l^{-\sigma+1}$ in (3b). The vertex with $i$ legs is proportional to $t /\langle l\rangle^{\sigma-1+i}$, where $t=\left|a_{0}\right|$. A typical contribution of a graph with $V$ vertices, $I$ internal lines, and $L$ loops to $\Gamma^{(2)}(0)$ is

$$
\frac{t^{V}}{\langle l\rangle\rangle^{\sum_{i}(\sigma-1+i)}} \xi^{2 I-L(d-1)},
$$

where $V_{i}$ is the number of vertices with $i$ legs and $\xi^{2 I-L(d-1)}$ the infrared divergence of the diagram. Since all graphs are infrared divergent for $d<3$, the superficial divergence $\xi^{2 I-L(d-1)}$ of the graph utilized in (4) is, in fact, the true divergence. Self-consistency requires that (4) be proportional to $\xi^{-2}$ for all graphs. Utilizing the topological relations $L=I-V+1$ and $\sum_{i} i V_{i}=2+2 I$ (for $\Gamma^{(2)}$ ) as well as the scaling relation $\beta_{s}=\frac{1}{2}(d-3) \nu$, this implies

$$
t \xi^{d-1}=\xi^{(3-d)(\sigma-1) / 2},
$$


so that $\nu=2 /[1+d-\sigma(3-d)]$. A similar calculation shows that $\nu / \Delta_{1}=2 /[2(d-1)-\sigma(3-d)]$ at the tricritical point. The relation for $\nu$ holds for critical wetting in the dimension interval $d_{0}<d<d_{1}$ and for the tricritical transition for $d_{1}<d<d_{2}$. The relation for $\Delta_{1}$ is correct for $d_{1}<d<d_{2}$. In these dimension intervals there is no shift in the MF values of the critical coupling constants. The generalization to higher-order transitions is straightforward.

As $d \rightarrow d_{1}^{+}, \Delta_{1} \rightarrow 0$. Similarly, as $d \rightarrow d_{0}^{+}, \nu \rightarrow \infty$. For $d<d_{1}, l^{-\sigma}$ is an irrelevant operator and the position of the tricritical point shifts to finite $a_{1}$. Similarly, for $d<d_{0}, l^{-\sigma+1}$ is irrelevant and the wetting transition occurs at finite $a_{0}$. This is verified explicitly for $d=2$ below. Note that $d_{0}$ is the same UCD found by Lipowsky at the complete wetting transition, i.e., above $T_{w}$ as coexistence is approached. $^{5}$

We have not been able to obtain the exponents for general $d$ for the multicritical transition $j$ for $d<d_{j-1}$. However, results for $d=2$ reported below lead to the following picture: For $d<d_{j-1}$ the critical exponents of all continuous transitions of order greater than or equal to $j$ are the same. Furthermore, for $d<d_{0}$ all transitions become continuous and belong to the same universality class, even those described by MF theory to be first order.

These predictions can be readily tested for $d=2$ using transfer matrix methods. ${ }^{23}$ The results of such a calculation are summarized here for the critical wetting transition:

$2>\sigma>1$, transition at $a_{0}=0$;

$$
2-\alpha_{s}=\sigma, \Delta=\sigma+1
$$

$3>\sigma>2$, transition at $a_{0}=0$,

$$
2-\alpha_{s}=\frac{2}{3-\sigma}, \Delta=\frac{3}{3-\sigma}, \frac{2-\alpha}{\Delta}=\frac{2}{3} ;
$$

$\sigma=3$, borderline case, transition at finite $a_{0}=a_{0}^{*}$,

$$
F_{s} \sim \exp \left(-t^{-1 / 2}\right),\langle l\rangle \sim \exp \left(t^{-1 / 2}\right) ;
$$

$\sigma>3$, transition at finite $a_{0}=a_{0}^{*}$,

$$
2-\alpha_{s}=2, \Delta=3, \frac{2-\alpha_{s}}{\Delta}=\frac{2}{3} \text {. }
$$

At the tricritical transition we find the following:

$2>\sigma>1$, transition at $a_{1}=0$,

$$
2-\alpha_{s}=\frac{2}{3-\sigma}, \Delta_{1}=\frac{2-\sigma}{3-\sigma}, \Delta=\frac{3}{3-\sigma}, \frac{2-\alpha_{s}}{\Delta}=\frac{2}{3} ;
$$

$\sigma=2$ borderline case, transition at finite $a_{1}=a_{1}^{*}$,

$$
2-\alpha_{s}=2, F_{s} \sim \exp \left[-\left(\Delta a_{1}\right)^{-1 / 2}\right] \text { for } a_{0}=0 ;
$$

$\sigma>2$, transition at finite $a_{1}=a_{1}^{*}$,

$$
2-\alpha_{s}=2, \Delta_{1}=1, \Delta=3, \frac{2-\alpha_{s}}{\Delta}=\frac{2}{3} \text {. }
$$

These results are in complete agreement with the general predictions discussed above.

As already mentioned, for $d<d_{0}$ we also expect there to be no first-order wetting transition. For $d>d_{0}, l^{1-\sigma}$ is a relevant operator and fluctuations are not strong enough to change the character of the transition as long as $a_{0}$ is finite. If $l^{1-\sigma}$ is irrelevant we have already seen that fluctuations change the phase diagram dramatically and shift the position of the wetting transition to finite $a_{0} . a_{0}=0$ is an infrared stable fixed point in this case and the presence of a barrier in $V(l)$ vanishing at least as fast as $l^{1-\sigma}$ should not affect the critical behavior. This is readily confirmed for $d=2$, where the problem reduces to a quantum-mechanical one. Given $V(l)$, the interfacial free-energy density is $-E$ in the thermodynamic limit, where $E$ is the lowest eigenvalue of the Schrödinger equation:

$$
\frac{d^{2} \Psi}{d l^{2}}-V(l) \Psi=-E \Psi
$$

In this approach, $\langle l\rangle$ is the ground-state expectation value of $l$. By definition, a bound state is one with finite $\langle l\rangle$. Consider now $V(l) \rightarrow a_{0} l^{1-\sigma}$ for large $l$, with $a_{0}>0$. The detailed behavior of $V$ for small $l$ is unimportant as long as it has attractive $(V<0)$ components and is bounded from below. Clearly, if $E<0(E>0)$ then $\langle l\rangle$ is finite (infinite). If for $E=0\langle l\rangle$ is finite, we have a first-order transition to infinite $\langle l\rangle$. On the other hand, the transition is continuous if $\langle l\rangle$ diverges with $E \rightarrow 0^{-}$.

Denoting the depth of the potential minimum by $V_{m}$, define $t \sim\left|V_{m}-V_{m}^{*}\right|$ where $E\left(V_{m}^{*}\right)=0$. Since the width of the minimum is finite, it is easily $\operatorname{seen}^{23}$ that $-E \sim t^{2}$ as $t \rightarrow 0^{+}$. To determine the order of the transition we may ask how $\langle l\rangle$ diverges with $t^{-1}$. Here, we report only the results $^{24}$ which are most easily characterized by the exponent $q$ in $\langle l\rangle \sim t^{-q}$. For $\sigma>3$ the transition is continuous with $q=1$. For $\sigma<3$ the transition is first order; i.e., $q=0$. The borderline case $\sigma=3$ displays richer structure, with $q=1$ for $a_{0}<\frac{3}{4}$, an amplitude dependent $q=\left(1+4 a_{0}\right)^{1 / 2}-3$ for $\frac{3}{4}<a_{0}<2$ and $q=0$ (but with divergent higher moments) for $a_{0}>2$. At the end points, where $q=0$ or 1 , there are additional $\ln t$ terms. The transition remains first order if $l^{1-\sigma}$ is a relevant operator but becomes continuous if $l^{1-\sigma}$ is irrelevant. Furthermore, the critical singularity in this case is the same as for short-range forces, in agreement with the general predictions discussed above.

After completing this work we received a paper by Fisher and $\mathrm{Huse}^{25}$ in which the critical wetting transition for longrange interactions was analyzed using a functional renormalization-group approach. Their results agree with ours.

R.L. thanks Professor M. E. Fisher for his hospitality at Cornell University and acknowledges support by a National Science Foundation grant. R.K.P.Z. wishes to thank Professor $H$. Wagner for his hospitality at the Universität München and the Alexander von Humboldt Foundation for its financial support. 
"Permanent address: Sektion Physik der Ludwig-MaximiliansUniversität München, D-8000 München, West Germany.

${ }^{1}$ R. Pandit, M. Schick, and M. Wortis, Phys. Rev. B 26, 5112 (1982).

2R. Lipowsky, D. M. Kroll, and R. K. P. Zia, Phys. Rev. B 27, 4499 (1983).

${ }^{3}$ E. Brézin, B. I. Halperin, and S. Leibler, Phys. Rev. Lett. 50, 1387 (1983).

${ }^{4}$ (a) M. P. Nightingale, W. F. Saam, and M. Schick, Phys. Rev. Lett. 51, 1275 (1983); (b) Phys. Rev. B 30, 3830 (1984).

${ }^{5}$ R. Lipowsky, Phys. Rev. Lett. 52, 1429 (1984).

${ }^{6}$ For a review, see D. E. Sullivan and M. M. Telo da Gama, in Fluid Interface Phenomena, edited by C. A. Croxton (Wiley, New York, in press); E. H. Hauge, in International Summer School on Fundamental Problems in Statistical Mechanics VI-Trondheim, Norway, June 18-29, 1984, edited by E. G. D. Cohen (North-Holland, Amsterdam, 1985).

${ }^{7}$ R. Lipowsky, Z. Phys. B 55, 335 (1984); 55, 345 (1984).

${ }^{8}$ V. Privman, J. Chem. Phys. 81, 2463 (1984).

${ }^{9}$ R. Lipowsky and D. M. Kroll, Phys. Rev. Lett. 52, 2303 (1984).

${ }^{10}$ D. M. Kroll and T. F. Meister, Phys. Rev. B 31, 392 (1985).

${ }^{11}$ S. Dietrich and M. Schick, Phys. Rev. B 31, 4718 (1985).

${ }^{12}$ C. Ebner, W. F. Saam, and A. K. Sen, Phys. Rev. B (to be published).
${ }^{13}$ This has also been pointed out by the authors of Refs. 4(b), 11, and 12 using the method of Ref. 5.

${ }^{14}$ M. E. Fisher, S. Ma, and B. G. Nickel, Phys. Rev. Lett. 29, 917 (1972).

${ }^{15}$ The case $\sigma=2$ can be handled in an analogous fashion and there is no change in the final result $(3 a)$ in this case.

${ }^{16}$ The arguments applied here are well known to investigators of nonlinear field equations. See R. Jackiw, Rev. Mod. Phys. 49, 681 (1977).

${ }^{17}$ E. Brézin, B. I. Halperin, and S. Leibler, J. Phys. (Paris) 44, 775 (1983).

${ }^{18}$ R. K. P. Zia, in Statistical and Particle Physics-Common Problems and Techniques, edited by K. C. Bowler and A. J. Mc Kane (Scottish Universities Summer School in Physics Publications, Edinburgh, 1984).

${ }^{19}$ N. Nakaniski and M. E. Fisher, Phys. Rev. Lett. 49, 1565 (1982).

${ }^{20}$ D. M. Kroll and R. Lipowsky, Phys. Rev. B 28, 6435 (1983).

${ }^{21}$ This can be shown explicitly for the case of complete wetting: R. Lipowsky, Phys. Rev. B 32, 1731 (1985).

22J. D. Weeks, Phys. Rev. Lett. 52, 2160 (1984).

${ }^{23}$ D. M. Kroll and R. Lipowsky, Phys. Rev. B 28, 5273 (1983).

${ }^{24}$ The details of this calculation will be presented elsewhere.

${ }^{25}$ D. S. Fisher and D. A. Huse (unpublished). 